\title{
Efficacité théorique d'un Indice de sélection sur collatéraux pour le foie gras de l'ole Landaise
}

\author{
R. ROUVIER *, A. VRILLON *, D. ROUSSELOT-PAILLEY ** et P. LARRUE ** \\ * I.N.R.A., Station d'Amélioration génétique des Animaux, \\ Centre de Recherches de Toulouse, \\ B.P. 12, F 31320 Castanet-Tolosan \\ ** I.N.R.A., Station expérimentale de l'Oie, Artiguères \\ B.P. 68, F 40002 Mont-de-Marsan
}

\begin{abstract}
Résumé
L'efficacité théorique d'un indice de sélection sur collatéraux pour améliorer le poids du foie gras de l'oie Landaise est étudiée. Sur $\mathrm{m}$ oisons disponibles par oie et saison de reproduction, $\mathrm{n}$ sont gavés et abattus pour servir de test des reproducteurs, $\mathrm{m}-\mathrm{n}$ animaux contemporains (dont la moitié de chaque sexe) sont candidats à la sélection. Cela revient à chercher l'optimum pour $\mathrm{n}$, de façon à maximiser le produit de l'intensité de sélection par la corrélation entre la valeur génétique additive et celle estimée par l'indice de sélection. Une solution sub-optimale est trouvée, pour un intervalle de génération de un an. Une solution pratique est proposée pour un noyau de sélection de faible effectif (160 oies).
\end{abstract}

Quelques auteurs (Boyer, 1969 ; STASKo et col., 1976), ont mis en évidence un antagonisme génétique entre production d'oisons et de foie gras chez l'oie, donné des généralités sur les paramètres génétiques des Palmipèdes gras et les objectifs de l'amélioration génétique. Pour l'Inter-Profession Palmipèdes gras, en France, les caractères prioritaires à améliorer, en vue d'accroître la rentabilité de la production d'oies grasses, sont le poids de foie obtenu en une durée de gavage courte et la qualité technologique du foie. Dans ce but, un schéma de sélection de l'oie à foie gras a été mis en place, avec constitution d'un noyau de sélection et test des performances foie gras, d'une part. D'autre part, une expérimentation en sélection de l'oie Landaise est effectuée à la Station Expérimentale de l'Oie d'Artiguères. Cela conduit notamment à établir puis appliquer un indice de sélection sur collatéraux pour le poids du foie gras. L'efficacité théorique de la méthode de sélection mise en œuvre doit être étudiée, de façon à rechercher les solutions optimales ou sub-optimales, et des solutions pratiques tenant compte des contraintes expérimentales ou économiques. Il nous a donc paru utile dans cette première note, de discuter l'efficacité théorique d'une méthode de sélection sur collatéraux pour le poids du foie gras chez l'oie Landaise. 


\section{Matériel animal et contraintes zootechniques}

L'oie Landaise de la Station d'Artiguères pond en saison naturelle de reproduction (janvier à juin), en moyenne, plus de 50 œufs en $1^{\text {re }}$ année et 60 œufs en $2^{\circ}$ année de ponte. Les reproducteurs sont conduits en parquets pédigree de 1 jars $\times 4$ oies. L'insémination artificielle ne peut être utilisée qu'à titre expérimental (avec du sperme frais). Elle n'est pas pratiquée en ferme. Les animaux sont gavés à l'âge de 5 mois, avant qu'ils ne soient en âge de produire des gamètes. Un animal gavé devient inapte à la reproduction. La mesure des caractères poids et qualité technologique du foie gras nécessite l'abattage de l'animal. Deux méthodes de sélection sont donc envisageables :

- une sélection sur collatéraux : les reproducteurs âgés de un an donnent une partie de leur descendance gavée pour le test des performances foie gras, l'autre partie constituant les animaux contemporains candidats à la sélection. L'intervalle minimum de génération est alors de un an;

- une sélection sur descendance : les reproducteurs mâles seraient testés et sélectionnés sur descendance à un an et utilisés à deux ans pour créer le progrès génétique et constituer la génération suivante. L'intervalle minimum de génération serait de deux ans.

\section{Estimation de la valeur génétique additive}

Les animaux servant au test et ceux candidats à la sélection sont issus d'un plan hiérarchique d'accouplement. Si $P_{i j k}$ est la variable aléatoire phénotypique attachée au $\mathrm{k}^{\mathrm{e}}$ descendant $\left(k=1\right.$ à $\left.n_{\mathrm{ij}}\right)$ de la $\mathrm{j}^{\mathrm{e}}\left(\mathrm{j}=1\right.$ à $\left.\mathrm{d}_{\mathrm{i}}\right)$ oie accouplée au $\mathrm{i}^{\mathrm{e}}$ jars (i $=1$ à s), l'on a :

$$
P_{i j k}=\mu+A_{i}+B_{i j}+E_{i j k}
$$

$\mu$ étant une constante, $A_{i}$ l'effet aléatoire du jars $i, B_{i j}$ celui de l'oie $i j, E_{i j k}$ celui du $\mathbf{k}^{\boldsymbol{\theta}}$ descendant de l'oie ij. Les effets aléatoires du modèle sont supposés être distribués normalement, respectivement $\mathrm{N}\left(\mathrm{O}, \gamma_{\mathrm{A}}\right), \mathrm{N}\left(\mathrm{O}, \gamma_{\mathrm{B}}\right), \mathrm{N}\left(\mathrm{O}, \gamma_{\mathrm{E}}\right)$, et être indépendants entre eux. La variance phénotypique se note $\sigma_{P}^{2}=\gamma_{A}+\gamma_{B}+\gamma_{E}$, et la variance génétique additive $\underset{\sigma_{\mathrm{G}}}{2}$.

\subsection{Indice de sélection sur collatéraux}

Celui-ci s'écrit :

$\hat{\mathrm{G}}_{\mathrm{ije}}=\beta_{1}\left(\mathrm{P}_{\mathrm{i} 1 .}-\mu\right)+\beta_{2}\left(\mathrm{P}_{\mathrm{i} 2 .}-\mu\right)+\ldots+\beta_{\mathrm{j}}\left(\mathbf{P}_{\mathrm{ij} .}-\mu\right)+\ldots+\beta_{\mathrm{d} 1}\left(\mathrm{P}_{\mathrm{idi} .}-\mu\right)$

Les coefficients de régression $\beta_{1}, \beta_{2}, \ldots ; \beta_{\mathrm{j}}, \ldots \beta_{\mathrm{d} 1}$ sont calculés de façon à minimiser la somme des carrés des écarts entre $\hat{G}_{\mathrm{ije}}$ et $\mathrm{G}_{\mathrm{ije}}$, ce qui revient à maximiser la correlation $\mathbf{R}_{\mathrm{G} \hat{G}}$ entre ces deux aléatoires (ROUVIER, 1977).

Pour étudier l'efficacité de cet indice, nous considérons un cas équilibré (d oies par jars, $n$ descendants contrôlés par oie), ce qui permet d'écrire : 


$$
\hat{G}_{i j e}=\frac{0,25 \sigma_{G}^{2}}{\gamma_{B}+\frac{\gamma_{E}}{n}}\left(P_{i j .}-P_{i . .}\right)+\frac{\frac{d+1}{4 d} \sigma_{G}^{2}}{\gamma_{A}+\frac{\gamma_{B}}{d}+\frac{\gamma_{E}}{n d}}\left(P_{i . .}-\mu\right)
$$

en utilisant la propriété de corrélation nulle entre les deux prédicteurs $\left(\mathbf{P}_{\mathrm{ij} .}-\mathbf{P}_{\mathbf{i} . .}\right)$ et $\left(\mathbf{P}_{\mathrm{i} . .}-\mu\right)$.

La précision de l'indice se mesure par la valeur de la corrélation ${ }_{\mathrm{C}} \mathrm{R}_{\mathrm{G}} \hat{\mathrm{G}}$ entre la valeur génétique additive $G$ et son estimée $\hat{G}$

$$
{ }_{\mathrm{C}^{2} \mathrm{G} \hat{\mathrm{G}}}=\frac{0,25 \frac{\mathrm{d}-1}{4 \mathrm{~d}} \sigma_{\mathrm{G}}^{2}}{\gamma_{\mathrm{B}}+\frac{\gamma_{\mathrm{E}}}{\mathrm{n}}}+\frac{\left(\frac{\mathrm{d}+1}{4 \mathrm{~d}}\right)^{2} \sigma_{\mathrm{G}}^{2}}{\gamma_{\mathrm{A}}+\frac{\gamma_{\mathrm{B}}}{\mathrm{d}}+\frac{\gamma_{\mathrm{E}}}{\mathrm{nd}}}
$$

\subsection{Indice de sélection sur descendance}

Pour raisonner de façon homogène, nous estimerons la valeur génétique additive $\mathrm{G}_{\mathrm{ij}^{\prime} \mathbf{e}^{\prime}}$ d'un futur descendant du jars $\mathrm{i}$, obtenu avec des oies de la population à améliorer, différentes des oies ayant donné des descendants contrôlés pour le test, mais supposées appartenir à la même population. Le phénotype prédicteur $P_{i . .}$ peut donc être centré par rapport à $\mu$.

$$
\begin{aligned}
& \dot{\mathrm{G}}_{\mathrm{ij}^{\prime} \mathrm{e}^{\prime}}=\frac{0,25 \sigma_{\mathrm{G}}^{2}}{\gamma_{\mathrm{B}}}\left(\mathrm{P}_{\mathrm{1..}}-\mu\right) \\
& \gamma_{A}+\frac{\gamma_{B}}{d}+\frac{\gamma_{E}}{\text { nd }} \\
& { }_{D^{2}} \mathbf{R}_{G \hat{G}}=\frac{0,25^{2} \sigma_{G}^{2}}{\gamma_{A}+\frac{\gamma_{B}}{d}+\frac{\gamma_{E}}{n d}}
\end{aligned}
$$

Les formules précédentes peuvent s'écrire en fonction des paramètres génétiques classiques, l'héritabilité $h^{2}$ et la corrélation intra classe $t=\frac{\gamma_{A}+\gamma_{B}}{\sigma_{P}^{2}}$ qui est la corré-

lation entre phénotypes de germains. Il suffit de poser $h^{2}=\frac{\sigma_{\mathrm{G}}^{2}}{\sigma_{\mathrm{P}}^{2}}=4 \frac{\gamma_{\mathrm{A}}}{\gamma_{\mathrm{P}}}$. 
La valeur maximum de ${ }_{D} R^{2}{ }_{G} \hat{G}$ est de 0,25 (donc 0,5 pour ${ }_{D} R_{G} \hat{\tilde{G}}$ si $n$ et $\mathrm{d}$ deviennent tous les deux très grands), ou si $d$ devient très grand $n$ étant au moins égal à 1 .

La valeur maximum de ${ }_{C^{2}} R_{G}^{2} \hat{G}$ si $n$ et $d$ deviennent tous les deux très grands est de $0,25\left(\frac{\gamma_{A}}{\gamma_{B}}+1\right)$. Si $\gamma_{B} \geqslant \gamma_{A}$, le maximum est ${ }_{C} R^{2}{ }_{G} \hat{G}=0,50$ et ${ }_{\mathrm{C}} \mathbf{R}_{\mathrm{G} \hat{\mathrm{G}}}=0,707$ lorsque $\gamma_{\mathrm{B}}=\gamma_{\mathrm{A}}$.

\section{Optimum pour l'espérance du progrès génétique}

L'espérance du progrès génétique par unité de temps s'écrit :

$$
\mathrm{E}(\Delta \mathrm{G})=\frac{1}{\theta} \mathrm{i} \cdot \mathrm{R}_{\mathrm{G} \hat{\mathrm{G}}} \cdot \sigma_{\mathrm{G}}
$$

$\theta$ étant l'intervalle de génération et i l'intensité de sélection. Dans le cas de sélection par troncature, $\mathrm{i}=\frac{\mathrm{z}}{\mathrm{p}}, \mathrm{z}$ étant l'ordonnée de la courbe normale réduite au point de troncature, $\mathrm{p}$ le pourcentage sélectionné qui permet de déterminer ce point de troncature.

La ponte étant saisonnée et annuelle, la situation d'accouplement 1 jars $\times 4$ oies étant imposée par la zootechnie de l'espèce, nous nous ramenons à une optimisation de $n$ (nombre d'oisons gavés par oie) de façon à maximiser $\mathrm{E}(\Delta \mathrm{G})$. Nous prenons comme paramètres zootechniques et de sélection :

$\theta=1$ an,

$\mathrm{m}=28$ oisons utilisables par oie et saison de reproduction,

$\mathrm{N}_{1}=\mathrm{N}_{2}$ mâles et femelles candidats à la sélection issus de chaque oie.

$$
\mathrm{N}_{1}=\mathrm{N}_{2}=\frac{\mathrm{m}-\mathrm{n}}{2}
$$

$P_{1}=\frac{1}{4 N_{1}}$ et $P_{2}=\frac{1}{N_{2}}$, taux de sélection respectivement des mâles et femelles

parmi les candidats à la sélection. L'on en déduit les intensités de sélection $\mathrm{i}_{1}$ et $\mathrm{i}_{2}$ dans les deux sexes, et la moyenne $i=\frac{i_{1}+i_{2}}{2}$. 
Les composantes de la variance du poids du foie ont été obtenues sur 340 oies des deux sexes gavées en 1980 (moyenne de poids du foie : $770 \mathrm{~g}$ ) : $\gamma_{\mathrm{A}}=2162 \mathrm{~g}^{2}$, $\gamma_{\mathbf{B}}=7578 \mathrm{~g}^{2}, \quad \gamma_{\mathbf{E}}=27991 \mathrm{~g}^{2}$. Ces paramètres sont assimilés aux paramètres théoriques de la population.

$$
\stackrel{2}{\sigma_{\mathrm{G}}}=4 \gamma_{\mathrm{A}}=8648 \mathrm{~g}^{2}, \text { et } \mathrm{h}^{2}=0,23
$$

\section{Résultats et discussion}

Le tableau 1 donne des valeurs de ${ }_{\mathrm{C}} \mathbf{R}_{\mathrm{G}} \hat{\mathrm{G}}$, $\hat{i}, \hat{\mathbf{i}}$. ${ }_{\mathrm{C}} \mathbf{R}_{\mathrm{G} \hat{\mathrm{G}}},{ }_{\mathrm{D}} \mathbf{R}_{\mathrm{G} \hat{\mathrm{G}}}$, pour $\mathrm{d}=4$ et $\mathrm{d}=10$, lorsque l'on fait varier $n$ de 2 à 26 par pas de 2 .

\section{TABleau 1}

Paramètres du gain génétique espéré, en fonction de $n$, nombre de descendants contrôlés par oie.

Parameters of expected genetic gain, in fonction of $n$, number of test animals per breeding goose.

Indice de sélection sur collatéraux.

Selection index according to the test of contemporaneous relatives.

$$
\mathrm{d}=4
$$

\begin{tabular}{|c|c|c|c|c|c|c|c|c|c|}
\hline $\mathrm{n}$ & 2 & 4 & 6 & 8 & 10 & 12 & 16 & 20 & 26 \\
\hline$N_{1}$ & 13 & 12 & 11 & 10 & 9 & 8 & 6 & 4 & 1 \\
\hline $\mathrm{N}_{2}$ & 13 & 12 & 11 & 10 & 9 & 8 & 6 & 4 & 1 \\
\hline${ }_{\mathrm{C}} \mathrm{R}_{\mathrm{G} \hat{\mathrm{G}}}^{2}$ & 0,36 & 0,42 & 0,44 & 0,46 & 0,47 & 0,48 & 0,48 & 0,49 & 0,49 \\
\hline i & 2,14 & 2,14 & 2,11 & 2,05 & 1,99 & 1,96 & 1,82 & 1,62 & 0,63 \\
\hline i. ${ }_{C} R_{G \hat{G}}$ & 0,77 & 0,90 & 0,93 & 0,94 & 0,94 & 0,94 & 0,87 & 0,80 & 0,31 \\
\hline \multicolumn{10}{|c|}{$d=10$} \\
\hline${ }_{\mathrm{C}} \mathbf{R}_{\mathrm{GG}}$ & 0,41 & 0,46 & 0,48 & 0,49 & 0,49 & 0,50 & 0,50 & 0,51 & 0,51 \\
\hline
\end{tabular}

Indice de sélection sur descendance.

Selecion index according to progeny testing.

\begin{tabular}{|c|c|c|c|c|c|c|c|c|c|}
\hline${ }_{D} R_{G \hat{G}}$ & $0,2 \overline{7}$ & 0,30 & 0,32 & 0,33 & 0,34 & 0,34 & 0,35 & 0,35 & 0,35 \\
\hline \multicolumn{10}{|c|}{$d=10$} \\
\hline${ }_{D} \mathbf{R}_{G \hat{G}}$ & 0,35 & 0,39 & 0,40 & 0,41 & 0,41 & 0,41 & 0,42 & 0,42 & 0,42 \\
\hline
\end{tabular}

$$
\mathrm{d}=4
$$


Le cas $\mathrm{d}=10$ a été considéré comme cas théorique. Il permet un accroissement plus important pour ${ }_{D} \mathbf{R}_{\mathrm{G} \hat{G}}$ que pour ${ }_{\mathrm{C}} \mathbf{R}_{\mathrm{G}} \hat{\mathrm{G}}$, indiquant l'intérêt d'inséminer plus de 4 oies par jars si l'on pratiquait une sélection sur descendance. Une solution suboptimale pour la sélection sur collatéraux consisterait à prendre $n=8$ ou $n=10$. Cela donnerait une espérance de gain génétique de $87 \mathrm{~g}$ de poids de foie. La valeur maximum de ${ }_{C} R_{G} \hat{G}$ est ici de 0,57 . L'on voit que ${ }_{C} R_{G \hat{G}}$ s'accroît lentement au-delà de $n=8$, et que la sélection sur collatéraux est plus précise que celle sur descendance. Il est évident qu'un intervalle de génération de un an s'accompagnerait de plus grand progrès génétique attendu par unité de temps.

La sélection sur collatéraux correspond au cas de la sélection expérimentale entreprise ou à celui d'une sélection en noyau de sélection dans lequel l'on cumulerait le progrès génétique en vue de diffusion en ferme par les animaux, leurs gamètes ou leurs œufs. Dans la sélection sur descendance, ce sont uniquement les jars sélectionnés qui permettraient la création et la diffusion du progrès génétique. L'on pourrait se ramener à la sélection sur collatéraux en tenant compte des performances des oies testées sur descendance. La position de notre problème est différente de celle de recherche de l'optimum du testage (RoBERTSON, 1957 ; NordsKog, 1959) lorsque la capacité totale de testage est fixée, de celle d'Ollivier (1974) qui recherche l'optimum entre intervalle de génération et intensité de sélection, de celle de Poujardieu \& Rouvier (1971) qui étudient l'optimum du plan d'accouplement dans la sélection combinée. Nous cherchons ici l'optimum entre $\mathbf{n}$ nombre de descendants de test, qui conditionne la précision de l'indice de sélection, et $\mathrm{N}_{1}, \mathrm{~N}_{2}$, nombres de descendants candidats à la sélection, qui conditionnent la valeur de l'intensité de sélection. Ce problème a été étudié par FREEDEN (1954) dans le cas du porc (cité par Ollivier, 1981). Une solution analytique pourrait être recherchée en utilisant l'approximation de C. SMITH (1969) qui permet d'exprimer l'intensité de sélection i en fonction du pourcentage sélectionné p. Si le noyau de sélection est d'effectif limité, la solution sub-optimale pour la sélection sur collatéraux peut avoir deux inconvénients, qui ne sont pas analysés ici, du fait des faibles valeurs de $\mathrm{p}$ : fort accroissement de la consanguinité (FoulleY, communication personnelle), imprécision sur le progrès génétique (Razungles, 1981). De plus, l'on peut être conduit, compte tenu des contraintes expérimentales, à rechercher une solution pratique. L'effectif de 4 descendants gavés par oie est une limite inférieure pour assurer des précisions équivalentes des estimées des composantes jar et oie de la variance (RoberTSON, 1959). Pour un noyau de sélection de 160 oies et 40 jars, et des tests en station, l'on pourrait contrôler en gavage 6 descendants de chaque oie et élever comme candidats à la sélection 8 fils et 8 filles de chaque oie. L'on aurait $\mathrm{E} \Delta \mathrm{G}=0,86 \sigma_{\mathrm{G}}$, ce qui représente $92 \mathrm{p}$. 100 de sa valeur pour la solution sub-optimum. Dans le cas d'un gavage en ferme, l'on pourrait tester 10 descendants par oie et renouveler un noyau de sélection de grand effectif à partir de 9 filles et 9 fils de chaque oie sélectionnée.

\section{Conclusion}

Cette première note n'envisage pas la question de la sélection de l'oie pour la production du foie gras dans sa globalité. L'objectif est, dans le cas où la sélection porte sur un caractère dont la mesure nécessite l'abattage de l'animal et où les candidats à la sélection peuvent être contemporains des animaux servant au test des 
reproducteurs, d'étudier l'efficacité théorique de la méthode de sélection utilisable. Pour une prolificité donnée, cela revient à chercher l'optimum entre le nombre d'animaux utilisés pour le test des reproducteurs et celui d'animaux conservés comme candidats à la sélection. L'intérêt de la méthode de sélection sur collatéraux de l'oie pour la production de foie gras réside dans le court intervalle de génération et les fortes intensités de sélection utilisables, avec une précision de l'indice de sélection quii s'accroît rapidement pour les petites valeurs du nombre d'animaux de test (lorsqu'elles passent de 2 à 12). Les charges expérimentales ou de sélection peuvent être diminuées par une solution pratique dont l'efficacité théorique est de peu inférieure à celle de la solution sub-optimale. Les limites au progrès génétique liées à la prise en compte d'autres caractères dans l'objectif de sélection, à la taille limitée du noyau de sélection devront être étudiées, expérimentalement et théoriquement. Dans le contexte dans lequel nous nous sommes situés, il semble qu'une sélection sur descendance serait moins efficace du fait d'une plus faible précision de l'indice de sélection et d'un plus grand intervalle de génération.

Reçu pour publication le 31 mars 1982.

\title{
Remerciements
}

Nous remercions J.L. Foulley pour sa lecture critique et ses suggestions qui ont permis d'améliorer le manuscrit.

\author{
Summary \\ Theoretical efficacy of a selection index on collaterals \\ for the fat liver of Landaise goose
}

Theoretical efficacy of a selection index to increase fat liver production of Landaise goose is studied. For $\mathrm{m}$ goslings per goose in each reproductive season, $\mathrm{n}$ are force feeded and slaughtered for the test of breeding animals, $\mathrm{m}-\mathrm{n}$ contemporaneous animals (half of each sex) are candidates for selection. We have to look for an optimum for $n$, number of animals of test, in order to maximise the product of selection intensity and correlation between additive genetic value and the one estimated by the selection index. A sub optimum solution is found, with a generation interval of one year. A practical solution is proposed for a nucleus of limited size (160 geese).

\section{Références bibliographiques}

BOYer J.P., 1969. Antagonisme génétique entre production de foie gras et production d'oisons. Aliment. Vie, 57, $\mathrm{n}^{\circ \mathrm{s}} 4,5,6,113-114$.

FrEEDEN H.T., 1954. Rate of genetic improvement in swine as influenced by the size and sex composition of test litters. Can. J. agric. Sci., 34, 121-130.

NoRDSKog A.W., 1959. Note on optimum group size for progeny tests. Biometrics, 15, 513-517. 
Ollivier L., 1974. Optimum replacement rates in animal breeding. Anim. Prod., 19, 257-271.

Ollivier L., 1981. Eléments de génétique quantitative. Ed. Masson, Paris.

Poutardieu B., Rouvier R., 1971. Optimisation du plan d'accouplement dans la sélection combinée. Ann. Génét. Sél. anim., 3 (4), 509-519.

Razungles J., 1981. Bases statistiques et génétiques des index de sélection. Variabilité de la réponse à la sélection. Thèse $3^{\mathrm{e}}$ cycle, 113 p., Université Paul Sabatier, Toulouse.

Robertson A., 1957. Optimum group size in progeny testing and family selection. Biometrics, 13, 442-450.

Robertson A., 1959. Experimental Design in the evaluation of genetic parameters. Biometrics, 15, 219-226.

Rouvier R., 1977. Mise au point sur le modèle classique d'estimation de la valeur génétique. Ann. Génét. Sél. Anim., 9 (1), 17-26.

Sмiтн C., 1969. Optimum selection procedures in animal breeding. Anim. Prod., 11, 433-442.

Stasko J., Grom A., Monachon G., 1976. Genetics and generalities on geese and ducks. Document à diffusion restreinte. Station expérimentale de l'Oie, Artiguères. 